\title{
Distribution of EGFR Mutations Commonly Observed in Primary Lung Adenocarcinomas in Pakistan as Predictors for Targeted Therapy
}

\author{
Zeeshan Ansar Ahmed, Tariq Moatter, Areeba Siddiqui, Shahid Pervez*
}

\begin{abstract}
Background: Acquired genetic alterations and presence of sensitizing mutations in the tyrosine kinase domain of EGFR and other signaling molecules have been found in different subsets of primary lung adenocarcinoma. The commonest EGFR mutations are small in frame deletions of exon 19 and a point mutation (L858R) in exon 21, having a combined occurrence of around $90 \%$. The objective of this study was to determine the frequency and types of EGFR mutations in primary lung adenocarcinomas in Pakistan. Materials and Methods: EGFR mutations in tumor samples were screened by multiplex real time PCR. Briefly, DNA from formalin fixed paraffin-embedded tissue was amplified with primers and probes specific to 43 different EGFR mutations in a Cobas z 480 instrument. The assay detects mutations in four exons (18-21) of the EGFR gene. Results: Out of 94 patients, 65 were males and 29 females with a M:F ratio of 2.2: 1. The median age was 62 years (range, 28 - 85 years). In our biopsy samples 70 (74\%) cases were of primary lung adenocarcinoma, whereas $24(26 \%)$ were confirmed metastatic adenocarcinoma of primary lung origin. EGFR mutation was positive in $29 \%$ of the patients. The highest frequency of L858R was observed in $48 \%$ of these, followed by deletion in exon 19 (44\%). In addition, other rare mutations such as compound G718X:S768I and insertions in exon 20 insertion were detected in approximately $4 \%$ of the patients. Conclusions: This study showed that Del 19 and L858R are the most frequent mutations in Pakistani lung adenocarcinoma patients and around $29 \%$ of the patients were found eligible for erlotinib therapy.
\end{abstract}

Keywords: EGFR - NSCLC - adenocarcinoma - tyrosine kinase - sensitizing mutations

Asian Pac J Cancer Prev, 15 (17), 7125-7128

\section{Introduction}

Lung cancer is a leading cause of malignancy associated human deaths, which is evident from its high mortality rate of 1.6 million (19.4\% of total) cancer deaths worldwide (Ferlay et al., 2013). It is more common in male and elderly group (Makay et al., 2006) risk factors include smoking, pollution, certain metals (chromium, cadmium), some organic chemicals and radiation. The risk of genetic susceptibility can contribute especially in young (Jemal et al., 2008; Ren et al., 2013).

Adenocarcinoma of lung is the commonest subset of lung cancer which is usually seen in nonsmokers and equally involves both males and females (Lindeman et al., 2013). It can present itself in early age as well. Fortunately, in the last decade due to major advances in our understanding of the pathogenesis and management of primary lung adenocarcinoma, new protocols of targeted treatment have emerged. Acquired genetic alterations and presence of sensitizing mutations in the tyrosine kinase domain of EGFR and other signaling molecules were found in different subsets of primary lung adenocarcinoma. Pharmacological agents targeting tyrosine kinase involved in growth factor receptor signaling like EGFR and ALK were developed and their discovery has changed the way, these malignancies are diagnosed and treated today (Rossi et al., 2013).

EGFR is a $170 \mathrm{KD}$ protein, which belongs to subclass 1 of transmembrane receptor tyrosine kinase superfamily. Its gene is located on chromosome 7p11.2 and has 28 exons (Salto-Tellez et al., 2011). It is normally expressed in various tissues of epithelial, mesenchymal and neural origin. Dysregulation of EGFR gene can lead to tumorogenesis. The over- expression of EGFR gene is seen in a variety of tumors including lung, head and neck, colon, pancreas, breast, bladder, kidney and gliomas (Wang et al., 2010; $\mathrm{Xu}$ et al., 2010). Around $60 \%$ of the primary lung adenocarcinomas are reported to have association with EGFR mutations; these sensitizing mutations are also found in non-smoker women of Asian origin. However, the frequency and types of mutations varied with the population examined (Liu et al., 2009; Wang et al., 2010; 
$\mathrm{Xu}$ et al., 2010).

The commonest EGFR mutations are small in frame deletion of exon 19 and point mutation (L858R) in exon 21 , having combined occurrence of around $90 \%$, whereas mutations in exon 18 and exon 20 accounted for 5\% respectively. The exon 19 and 21 mutations are found to be highly sensitive to reversible TK inhibitors. In contrast, exon 18 and 20 are less sensitive or insensitive to reversible TK inhibitors (Hanif et al., 2009; Ma et al., 2010). In Pakistan like rest of the world lung cancer prevalence is high $(15 \%)$ and primary lung adenocarcinoma constitute about $40-45 \%$ of primary lung cancer (Hanif et al., 2009).

The aim of the present study was to identify spectrum of EGFR mutations in Pakistani lung cancer (adenocarcinoma) patients, and associate them with clinical and histopathological parameters.

\section{Materials and Methods}

In this cross sectional study, a total of 94 patients, who approached for EGFR mutation analysis to the Molecular Pathology Section, Department of Pathology and Microbiology, Aga Khan University Hospital were enrolled consecutively from June 2013 to January 2014. The patients were confirmed for the presence of adenocarcinoma of lung on the basis of morphological and immunohistochemical workup. Typical immunohischemical profile of these showed CK 7 positive, TTF1 positive and CK5/6, CK20 and p63 negative. The source of biopsy specimen included primary lung lesions, metastatic lymph node, mediastinal, pleural and peritoneal tissue representing distant or direct metastases. (Figure 1 and 2).

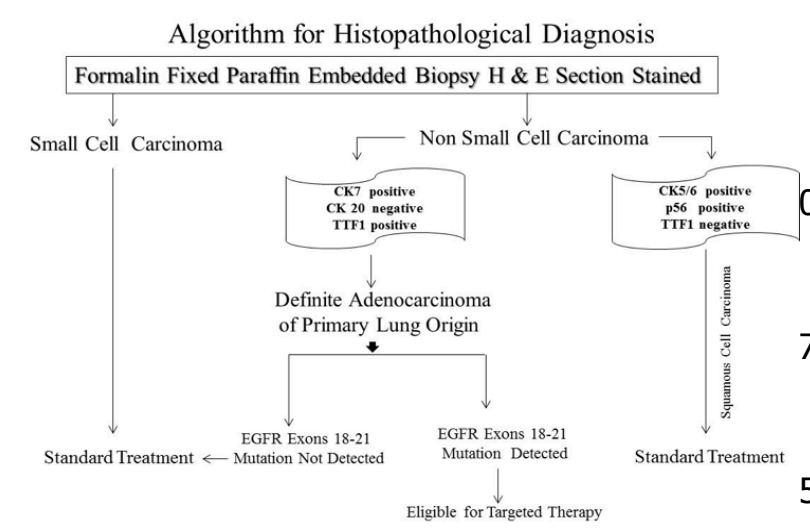

Figure 1. Algorithm for Histopathological Diagnosis

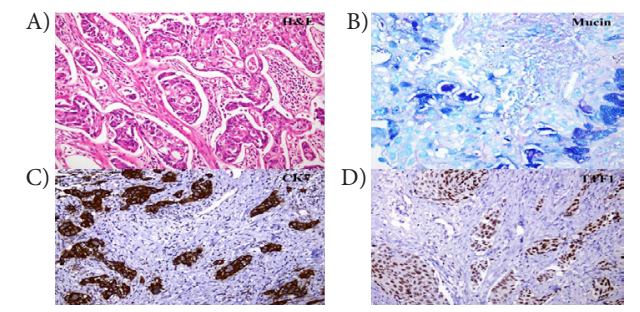

Figure 2. Photomicrographs of Primary Lung Adenocarcinoma. A) H \& E showing morphology of typical primary lung adenocarcinoma with glandular differentiation; B) Mucin is demonstrated; C) Cytokeratin 7 stained tumor cells strongly and diffusely; D) TTF1 nuclear positivity in tumor cells
DNA extraction and realtime PCR assay

For the identification of EGFR mutations, depending upon the size of the tissue, $2-5$ sections of $5 \mu \mathrm{M}$ thickness of FFPE specimen were cut and placed in $1.5 \mathrm{ml}$ microfuge tubes. DNA extraction and multiplex PCR was performed using Cobas EGFR mutation test kit and the Cobas z480 Realtime PCR instrument (Roche Molecular Systems, Branchburg, NJ, USA), following the standard package insert protocol. The extracted DNA was quantified by NanoDrop Analyzer (Wilmington, DE, USA) and a total of 150ng DNA was used for amplification. Cobas EGFR Mutation Test uses allele specific polymerase chain reaction (AS-PCR) and can detect 43 different mutations in the four exons (18-21) of EGFR gene, including several point mutations, deletions and insertions. For the assessment of quality parameters and validity of the run a mutant positive and negative controls were included in every batch of the PCR assay. Analysis was performed with the EGFR Analysis Package Software V.1.0 (Roche Molecular Systems).

\section{Statistical analysis}

Data were summarized and presented using appropriate descriptive statistics. Chi square test was performed to assess differences in the proportion of EGFR mutations across age, gender and disease severity as categories. Statistical analysis were performed using SPSS version 19.0 for Windows (IBM Inc., Chicago, IL, USA) and p value of $<0.05$ was set for level of significance.

\section{Results}

In this study, Formalin fixed paraffin embedded specimens of primary lung adenocarcinoma were analyzed for mutations in the exon 18-21 of EGFR gene. Out of 94 patients, 65 were males and 29 females with M: F ratio of 2.2: 1 . The median age of the patients was 62 years (range, $28-85$ years). In our biopsy samples $70(74 \%)$ cases were primary lung adenocarcinoma, whereas 24 (26\%) were metastatic adenocarcinoma samples, confirmed to be metastases from lung.

EGFR mutations were positive in $29 \%$ of the patients. As shown in Table 2, the highest frequency of L858R was observed in $48 \%$ of the patients, followed by deletion in exon 19 (44\%). In addition, other rare mutations such as compound G718X:S768I and insetions in exon 20 insertion were detected in approximately $4 \%$ of the patients.

Adenocarcinoma of lung in our patients was further categorized into two; primary lung adenocarcinoma

Table 1. Description of Cases Used for EGFR Mutational Analysis

\begin{tabular}{lccc}
\hline Histology of lung carcinoma & $\begin{array}{c}\text { Total } \\
\text { cases }\end{array}$ & $\begin{array}{c}\text { No. of EGFR } \\
\text { mutation }\end{array}$ & $\begin{array}{c}\text { Exon } \\
\text { mutation }\end{array}$ \\
\hline Primary adenocarcinoma & 70 & 20 & $18,20,21$ \\
Well differentiated & 40 & 10 & - \\
Moderately differentiated & 20 & 7 & - \\
Poorly differentiated & 10 & 3 & - \\
Metastatic adenocarcinoma of primary lung origin & \\
& 24 & 7 & $18,19,20,21$
\end{tabular}


Table 2. Patient Characteristics and Mutational Analyses of 94 Pakistani Lung Adenocarcinoma Cases

\begin{tabular}{|c|c|c|c|c|c|c|}
\hline & & \multirow[t]{2}{*}{ Total cases } & \multicolumn{2}{|c|}{ EGFR mutation } & \multirow{2}{*}{\multicolumn{2}{|c|}{ Mutation types number }} \\
\hline & & & Positive & Negative & & \\
\hline Cases & & $\mathrm{N}=94$ & $\mathrm{~N}=27(29 \%)$ & $\mathrm{N}=67(71 \%)$ & $\begin{array}{l}\text { Del (19exon) } \\
\text { Exon } 20 \\
\text { L858R } \\
\text { S768I/G719X }\end{array}$ & $\begin{array}{r}12 \\
1 \\
13 \\
1\end{array}$ \\
\hline Gender & $\begin{array}{l}\text { Male } \\
\text { Female } \\
\text { Age (years) (Median/Range) }\end{array}$ & $\begin{array}{c}65 \\
29 \\
62 \pm 12 / 28-85\end{array}$ & $\begin{array}{l}14(60 \%) \\
13(40 \%)\end{array}$ & & & \\
\hline Histological type & $\begin{array}{l}\text { Primary lung Adenocarcinoma } \\
\text { Metastatic Adenocarcinoma primary lung origin }\end{array}$ & $\begin{array}{l}\mathrm{N}=70 \\
\mathrm{~N} \quad \mathrm{~N}=24\end{array}$ & $\begin{array}{l}\mathrm{N}=20(28 \%) \\
\mathrm{N}=7(29 \%)\end{array}$ & & $\begin{array}{l}\text { del(19exon) } \\
\text { Exon } 20 \text { ins } \\
\text { Exon21L858R }\end{array}$ & $\begin{array}{r}9 \\
1 \\
10\end{array}$ \\
\hline Total No. of cases & & $\mathrm{N}=94$ & & & $\begin{array}{l}\text { del(19exon) } \\
\text { Exon21L858R } \\
\text { Compound mutation } \\
\text { \{Exon20S768I, Exon20G719X\} }\end{array}$ & $\begin{array}{l}3 \\
3 \\
1\end{array}$ \\
\hline
\end{tabular}

samples and metastatic adenocarcinoma samples of primary lung origin. The primary adenocarcinoma form was further classified into well, moderate and poorly differentiated subtypes. The frequency of EGFR mutations in these subsets of the adenocarcinoma was $35 \%$ and $25 \%$ respectively. EGFR mutations were more frequently found in well differentiated subtypes as presented in Table 1. In addition, no preponderance of gender on mutation status was noted, which may require large data set to avoid selection bias.

\section{Discussion}

Clinical trials and published data show that EGFR mutation analysis is highly desirable on primary lung adenocarcinoma as patients significantly benefit from specific tyrosine kinase inhibitors. Therefore to choose best option for these patients the analysis is a prerequisite.

Salto-telkz et al (2011) approximated that 30\%-80\% of lung adenocarcinoma patients are tested for EGFR mutation in East Asia. In this part of the world EGFR testing in primary lung adenocarcinoma is very limited. Reason includes lack of testing facilities and high cost. Our institute is the first in the 6th most populated countries of the world with an estimated population of 186 million, hence is the first report of mutational analyses from this country.

The worldwide occurrence of lung cancer is approximately $25 \%$ in lifelong nonsmokers (Ferlay et al., 2012) in which around 30 to $40 \%$ are from Asian countries (Xiao et al., 2011). In both male and female nonsmokers the mortality rates from primary lung adenocarcinoma are 17.1 and $14.7 / 1000$ person/year respectively. The proportion of primary lung adenocarcinoma in nonsmokers is indeed one of the most common causes of cancer-related death (Parkin et al., 2005).

Ma et al (2010) reported high prevalence of EGFR mutation in primary lung adenocarcinoma from northeast (China, Japan, Korea) and part of Southeast Asia (excluding India) nonsmokers, predicting high sensitivity to TKI. Another study from Indian reported around $30 \%$ of primary adenocarcinoma of lung belonged to subgroup of NSCLC, their data matched with other global study where EGFR mutation rate was 1.7 times more in female verses 1.5 times more in Indian cohort. Their prevalence of EGFR mutation being matched with East Asian data of 27-60\%.

Noronha et al. (2013) recently showed, data shows $96 \%$ of patients who primary adenocarcinoma of lung in which majority was nonsmoker with significant proportion of females (48\%), 35\% of these patients were found to harbor an EGFR mutation.

Regarding the types of EGFR mutation detected in their study, $74 \%$ of patients were noted to have deletion (exon 19), 23\% patients had L858R point mutation (exon 21 ) and only $2.5 \%$ had G719C point mutation exon 18.

Published literatures strongly suggest an association between grade, age, ethnicity and EGFR positivity. Multiple studies have also shown improved response and better quality of life along with high survival in the subset of EGFR positive patients with response to TKIs (Salto-Tellez et al., 2011).

Our study for the first time reveals information regarding EGFR mutational prevalence in Pakistani population, where 94 lung cancer patients of primary lung adenocarcinoma, specimens received from every part of Pakistan was analyzed, using the most sensitive diagnostic technique. As compared to other studies which showed that adenocarcinoma is more common in female (Wang et al., 2011), our study could not comment yet because of small size of the cases tested. Overall prevalence of EGFR mutation slightly matched with reported Asian data which ranges between 27-60\% in various studies (Cohen et al., 2010).Another similarity in our data with Western and Asian data is the highest mutation rate of $48 \%$ noted for L858R (exon 21) followed by deletions of exon 19 (44\%) of EGFR mutant isoform.

Other exons mutations were also identified like compound G718X (exon18) with S768I (exon20) and isolated exon 20 insertions seen in $4 \%$ of cases. In published data these are rare mutations and also resistant to targeted therapy but chemotherapy is another optional choice for these rare cases. The current recommendation for testing EGFR mutation is limited to adenocarcinoma of lung or nonsquamous lung cancer with adenocarcinoma component. The significant finding of positive EGRF mutation is high among patients of East Asian origin and female nonsmokers, however it is recognized that the immunohistopathological feature alone cannot be used to select for EGFR mutation analysis (Lindeman et al., 2013). 
The benefit of EGFR positive lung adenocarcinoma have better response rates when treated with TKI than with chemotherapy (Maemondo et al., 2010; Zhou et al., 2011; Han et al., 2012; Sequist et al., 2013) For assessment of treatment response and emergence of resistance in our patients we need larger studies with clinical data and follow up would determine impact of TKIs treatment of EGFR sensitizing lung adenocarcinoma.

In conclusion, L858R (exon 21) and deletion 19 exon were most frequent mutations in primary lung adenocarcinoma patients of Pakistani origin. These observations have led to changes in the overall treatment strategies for lung adenocarcinoma. Therefore, a genetic testing before the treatment is considered essential for primary adenocarcinoma of lung in order to select the appropriate treatment option according to the patient's molecular characteristics.

\section{References}

Cohen V, Agulnik JS, Ang C, et al (2010). Epidermal growth factor receptor mutations detected by denaturing high performance liquid chromatography in non-small cell lung cancer: impact on response to therapy with epidermal growth factor receptor-tyrosine kinase inhibitors. Cancer, 116, 4215-432.

Ferlay J, Soerjomataram I, Ervik M, et al (2012). Cancer Incidence and Mortality Worldwide: IARC Cancer Base. GLOBOCAN, 1.0, No. 11

Ferlay J, Shin HR, Bray F, et al (2010). Estimates of world-wide burden of cancer in 2008: GLOBOCAN 2008. Int J Cancer, 127, 2893-917.

Hanif M, Zaidi P, Kamal S, Hameed A (2009). Institution-based Cancer Incidence in a Local Population in Pakistan: Nine Year Data Analysis. Asian Pac J Cancer Prev, 10, 227-30.

Han JY, Park K, Kim SW, et al (2012). First-SIGNAL: first-line single-agent iressa versus gemcitabine and cisplatin trial in never-smokers with adenocarcinoma of the lung. J Clin Oncol, 30, 1122-8.

Jemal A, Thun MJ, Ries LA, et al (2008). Annual report to the nation on the status of cancer, 1975-2005, featuring trends in lung cancer, tobacco use, and tobacco control. J Natl Cancer Inst, 100, 1672-94.

Liu H, Li Y, Chen G, et al (2009). Detection and its clinical significance of EGFR gene mutation and gene amplification in 187 patients with non-small cell lung cancer. Zhongguo Fei Ai Za Zhi, 12, 1219-47.

Lindeman NI, Cagle PT, Beasley MB, et al (2013). Lung Cancer Molecular Testing Guidelines. Arch Pathol Lab Med, 137, 828-60.

Lindeman NI, Cagle PT, Beasley MB, et al (2013). Molecular testing guideline for selection of lung cancer patients for EGFR and ALK tyrosine kinase inhibitors: guideline from the College of American Pathologists, International Association for the Study of Lung Cancer, and Association for Molecular Pathology. J Thorac Oncol, 8, 823-59.

Mackay J, Jemal A, Lee NC, et al (2006). The Cancer Atlas. Atlanta: American Cancer Society.

Ma BBY, Hui EP, Mok TSK (2010). Population-based differences in treatment outcome following anticancer drug therapies. Lancet Oncol, 11, 75-84.

Maemondo M, Inoue A, Kobayashi K, et al (2010). Gefitinib or chemotherapy for non-small-cell lung cancer with mutated EGFR. $N$ Engl J Med, 362, 2380-8.

Mitsudomi T, Morita S, Yatabe Y, et al (2010). Gefitinib versus cisplatin plus docetaxel in patients with non-small-cell lung cancer harboring mutations of the epidermal growth factor receptor (WJTOG3405): an open label, randomized phase 3 trial. Lancet Oncol, 11, 121-8.

Noronha V, Prabhash K, Thavamani A, et al (2013). EGFR Mutations in Indian Lung Cancer Patients: Clinical Correlation and Outcome to EGFR Targeted Therapy. PLoS ONE, 8, 61561-69

Parkin DM, Bray F, Ferlay J, Pisani P (2005). Global cancer statistics, 2002. CA Cancer J Clin, 55, 74-108.

Rosell R, Carcereny E, Gervais R, et al (2012). Erlotinib versus standard chemotherapy as first-line treatment for European patients with advanced EGFR mutation-positive nonsmallcell lung cancer (EURTAC): a multicentre, open-label, randomized phase 3 trial. Lancet Oncol, 13, 239-46.

Rossi A, Pasquale R, Esposito C, Normanno N (2013). Should epidermal growth factor receptor tyrosine kinase inhibitors be considered ideal drugs for the treatment of selected advanced non-small cell lung cancer patients? Cancer Treatment Reviews, 5, 489-97.

Ren YW, Yin ZH, Wan Y, et al (2013). P53 Arg72Pro and MDM2 SNP309 polymorphisms cooperate to increase lung adenocarcinoma risk in Chinese female non-smokers: a case control study. Asian Pac J Cancer Prev, 14, 5415-20.

Salto Tellez M, Tsao MS, Shih JY, et al (2011): Clinical and testing protocols for the analysis of epidermal growth factor receptor mutations in East Asian patients with non-small cell lung cancer: a combined clinical-molecular pathological approach. J Thorac Oncol, 6, 1663-9.

Sequist LV, Yang JC, Yamamoto N, et al (2013). Phase III study of afatinib or cisplatin plus pemetrexed in patients with metastatic lung adenocarcinoma with EGFR mutations. $J$ Clin Oncol, 31, 3327-34.

Wang Y, Liu H, Chen J (2010). The predictive value of EGFR status in non-small cell lung cancer patients treated with EGFR-TKIs. Zhongguo Fei Ai Za Zhi, 13, 375-9.

Xiao H, Ding J, Gao S, Yang S, Huang Y (2011). Never smokers with lung cancer: analysis of genetic variants. Asian Pac J Cancer Prev, 12, 2807-9.

Xu Y, Chen L, Tian Q, et al (2010). Application of epidermal growth factor receptor tyrosine kinase inhibitor as the firstline therapy in patients with advanced non-small cell lung cancer. Zhongguo Fei Ai Za Zhi, 13, 48-53.

Zhou C, Wu YL, Chen G, et al (2011). Erlotinib versus chemotherapy as first-line treatment for patients with advanced EGFR mutation-positive non-small-cell lung cancer (OPTIMAL, CTONG-0802): a multicentre, openlabel, randomised, phase 3 study. Lancet Oncol, 12, 735-42. 